\title{
Preoperative endoscopic biliary stenting before pancreatico duodenectomy: Does timing matter?
}

\section{Abu Bakar Hafeez Bhatti ( $\nabla$ abubakar.hafeez@yahoo.com )}

Shifa International Hospital Islamabad https://orcid.org/0000-0002-4875-603X

\section{Roshni Zahra Jafri}

Shifa International Hospital Islamabad

\section{Kashif Khan}

Federal Government Polyclinic Hospital Islamabad

\section{Faisal Saud Dar}

Shifa International Hospital Islamabad

\section{Research article}

Keywords: Pancreaticodoudenectomy, morbidity, mortality, endoscopic stenting

Posted Date: June 29th, 2020

DOl: https://doi.org/10.21203/rs.3.rs-37972/v1

License: (9) This work is licensed under a Creative Commons Attribution 4.0 International License. Read Full License 


\section{Abstract}

\section{Background}

Role of preoperative biliary stenting (PBS) before pancreaticodoudenectomy (PD) in patients with obstructive jaundice is debatable. The objective of the current study was to assess outcomes after PD in patients who underwent upfront surgery or PBS and determine the impact of stent to surgery duration on outcomes after PD.

\section{Methods}

We reviewed 147 patients who underwent PD between 2011 and 2019. Patients were grouped based on whether they underwent upfront surgery $(\mathrm{N}=76)$ or PBS $(\mathrm{N}=71)$. We further assessed outcomes based on stent to surgery interval $<4$ weeks or $>4$ weeks. We looked at 30 and 90 day morbidity and mortality rates in these patients.

\section{Results}

A significant increase in wound infections ( $7 \%$ vs $25 \%)(P=0.003)$, overall infectious complications $(22.5 \%$ vs $38.1 \%)(P=0.04)$, re admissions ( 0 vs $10.5 \%)(P=0.005)$ and hospital stay (9 vs 10 days $)(P=0.006)$ was seen in the PBS group. There was no significant difference in 30 day mortality ( $2.8 \%$ vs $6.5 \%)(P=0.4)$. When compared with upfront surgery group, patients with stent to surgery duration $>4$ weeks had higher rates of wound infection ( $7 \%$ vs $29 \%)(P=0.009)$, sepsis $(11.2 \%$ vs $29 \%)(P=0.02)$, overall infectious complications ( $22.5 \%$ vs $45.1 \%)(P=0.02)$, re admissions ( 0 vs $12.9 \%)(P=0.007)$ and hospital stay (9 vs 10 days $)(P=0.03)$. The lowest rate of infectious complications was seen when $P D$ was performed within

2 weeks $(22.2 \%)$ or $6-8$ weeks $(12.5 \%)$ after stenting. None of the patients with stent-surgery duration $<2$ weeks developed sepsis.

\section{Conclusions}

PBS appears to increase infection related morbidity after PD. In patients with PBS, low morbidity is seen with early ( 2 weeks) and delayed PD (6-8weeks).

\section{Introduction}

Role of preoperative biliary stenting(PBS) in jaundiced patients with peri-ampullary malignancy is debatable. It was introduced in 1960s to reduce the risk of infections and anastomotic leaks after pancreaticodoudenectomy (PD) $[1,2]$. It is assumed that PBS corrects physiological disturbances due to hyperbilirubinemia in malignant biliary obstruction. However, improvement in postoperative outcomes after PD has not been consistently demonstrated in clinical practice [3-5]. In fact, a direct relationship between rate of postoperative infections and morbidity has been shown in patients with PBS [6, 7]. This has not only challenged the conventional approach of routine PBS but raises questions regarding the 
timing of surgery after biliary drainage, and the impact of delay in surgical intervention on postoperative outcomes.

Data remains limited and conflicting, with some reports demonstrating improved outcomes with a short stent to surgery interval ( $<2$ weeks) while others showing inferior outcomes with a stent to surgery duration of $<4$ weeks $[8,9]$.

The objective of the current study was to determine postoperative outcomes in patients who did and did not have PBS and assess the impact of time interval between stenting and surgery.

\section{Methods}

A total of 157 PDs were performed between January 2011 and July 2019. Adult patients(age > 18 years), who underwent endoscopic retrograde cholangio pancreatography (ERCP) stenting, with a minimum follow up of 3 month after surgery were included $(N=147)$.

We routinely perform PBS in patients with cholangitis or total bilirubin (TB) $>10 \mathrm{mg} / \mathrm{dl}$. However, many patients are referred from other centers for surgery after PBS. As a result, patients who undergo PD have variable total bilirubin cutoffs before PBS. For the purpose of this study, time between last PBS and PD was used to determine stent to surgery interval. We excluded patients who underwent percutaneous transhepatic cholangiography (PTC), combined ERCP/PTC or surgical bypass before PD. All patients included in the study had good functional status (Eastern cooperative oncology group 0 and 1 ) and underwent plastic stent placement. Details pertinent to patient selection, operative procedure, and post operative care have been detailed elsewhere $[10,11]$.

For this study, all patients with positive wound cultures or culture positive abdominal collections were considered to have wound infection or infected abdominal collections respectively. Sepsis was defined based on previously defined criteria [12] or positive blood cultures. Post operative pancreatic fistula (POPF) was graded based on International study group on pancreatic fistula (ISGPF) guidelines [13].

Primarily we looked at 90 day morbidity and mortality in stented and non-stented patients. We also compared outcomes based upon stent to surgery interval. Patients in the short duration stent to surgery group underwent surgical resection in $\leq 4$ weeks of PBS while the long duration group had PD $>4$ weeks after stenting. Clavien Dindo grading was used to assess severity of complications [14]. Mean and Median were reported for interval data based on normal distribution. For statistical significance, $t$ test and Mann Whitney $U$ test was used. For categorical variables, Chi Square and Fischer test was used to determine significance. A P value $<0.05$ was considered statistically significant. All analysis was performed on SPSS version 20. The hospital ethics committee approved the study.

\section{Results}

\section{Patient characteristics}


Mean age was $57.2 \pm 12.6$ years. Median follow up was $11.2(0.2-79)$ months. Median TB before intervention in the PBS and upfront surgery group was $5.3(0.2-49.8)$ and $4.5(0.2-24.8) \mathrm{mg} / \mathrm{dl}(\mathrm{P}=$ $0.227)$. Median TB at the time of surgery was $1.7(0.2-31) \mathrm{mg} / \mathrm{dl}$ and $4.5(0.2-24.8) \mathrm{mg} / \mathrm{dl}(P=0.087)$ in the PBS and upfront surgery groups respectively a shown in Table 1. Four patients did not show any improvement in jaundice after PBS and underwent PD despite high TB levels. Reasons included failure to retrieve occluded stent $(n=2)$ and upper GI bleed $(n=2)$.

Table 1

Preoperative characteristics in upfront surgery and preoperative biliary stenting groups

\begin{tabular}{|c|c|c|c|c|c|}
\hline & \multicolumn{2}{|c|}{$\begin{array}{l}\text { Preoperative endoscopic } \\
\text { drainage } \\
(n=76)\end{array}$} & \multicolumn{2}{|c|}{$\begin{array}{l}\text { Upfront } \\
\text { surgery } \\
(n=71)\end{array}$} & $\begin{array}{l}\mathrm{P} \\
\text { value }\end{array}$ \\
\hline Age $($ mean $\pm S D)$ & \multicolumn{2}{|c|}{$57.9 \pm 13.5$} & \multicolumn{2}{|c|}{$56.4 \pm 11.7$} & 0.4 \\
\hline Gender (Male) & 51 & 67.1 & 47 & 66.1 & 0.9 \\
\hline $\begin{array}{l}\text { Pre operative total bilirubin } \\
\text { (median/range) }\end{array}$ & \multicolumn{2}{|c|}{$1.7(0.2-31)$} & \multicolumn{2}{|c|}{$4.5(0.2-24.8)$} & 0.087 \\
\hline Benign etiology & 10 & 13.1 & 5 & 7 & 0.2 \\
\hline
\end{tabular}

\section{Upfront surgery versus PBS}

Table 2 demonstrates comparison of outcomes in patients with upfront surgery versus PBS. A significant increase in the rates of wound infection $(5 / 71[7 \%]$ versus $19 / 76[25 \%])(P=0.003)$, overall infectious complications $(16 / 71[22.5 \%]$ versus $29 / 76[38.1 \%])(P=0.04)$, readmission rates $(0$ versus $8 / 76[10.5 \%])(P$ $=0.005)$ and overall hospital stay $9(6-70)$ and $10(7-28)(P=0.006)$ was seen in the PBS group. 
Table 2

Comparison of post operative outcomes in upfront surgery versus preoperative biliary stenting group

\begin{tabular}{|c|c|c|c|c|c|}
\hline \multirow[b]{2}{*}{30 day mortality } & \multicolumn{2}{|c|}{$\begin{array}{l}\text { Upfront surgery } \\
(n=71)\end{array}$} & \multicolumn{2}{|c|}{$\begin{array}{l}\text { Preoperative drainage } \\
(n=76)\end{array}$} & \multirow{2}{*}{$\begin{array}{l}\text { P value } \\
0.4\end{array}$} \\
\hline & 2 & 2.8 & 5 & 6.5 & \\
\hline 90 day morbidity & 39 & 54.9 & 47 & 61.8 & 0.3 \\
\hline 90 day mortality & 3 & 4.2 & 8 & 10.5 & 0.2 \\
\hline Grade B/C post operative pancreatic fistula & 3 & 4.3 & 5 & 6.5 & 0.7 \\
\hline Gastroparesis & 7 & 9.8 & 4 & 5.2 & 0.3 \\
\hline Wound infection & 5 & 7 & 19 & 25 & 0.003 \\
\hline Sepsis & 8 & 11.2 & 17 & 22.3 & 0.07 \\
\hline Infected Intra abdominal collections & 9 & 12.6 & 6 & 7.8 & 0.4 \\
\hline Overall infectious complications & 16 & 22.5 & 29 & 38.1 & 0.04 \\
\hline Sterile Intra abdominal collections & 6 & 8.4 & 9 & 11.8 & 0.5 \\
\hline Re exploration & 3 & 4.2 & 1 & 1.3 & 0.3 \\
\hline Re admission rate & 0 & - & 8 & 10.5 & 0.005 \\
\hline Length of stay (days) & \multicolumn{2}{|c|}{$9(6-70)$} & \multicolumn{2}{|c|}{$10(7-28)$} & 0.006 \\
\hline Surgery duration (minutes) & \multicolumn{2}{|c|}{$470(270-720)$} & \multicolumn{2}{|c|}{$477(240-720)$} & 0.7 \\
\hline Blood loss (milliliters) & \multicolumn{2}{|c|}{$500(20-5000)$} & \multicolumn{2}{|c|}{$600(150-3000)$} & 0.85 \\
\hline
\end{tabular}

\section{Upfront surgery versus short and long stent to surgery interval}

Median TB before intervention in the upfront surgery group, short stent to surgery duration group and long stent to surgery duration group was $4.5 \mathrm{mg} / \mathrm{dl}, 4.9 \mathrm{mg} / \mathrm{dl}$ and $6 \mathrm{mg} / \mathrm{dl}(P=0.774)$. Median preoperative bilirubin was 4.5, 2.2 and $1.2 \mathrm{mg} / \mathrm{dl}(P=0.039)$ as shown in Fig. 1. Table 3 demonstrates results of subgroup analysis between upfront surgery and PBS groups with regards to stent to surgery duration. Wound infection, rate of re admission and length of hospital stay was significantly increased in the short stent to surgery duration group when compared with no stenting group. Outcomes were significantly worse for all outcome variables in the long stent to surgery duration group. 
Table 3

Comparison of outcomes in patients with upfront surgery versus stent to surgery duration $\leq 4$ weeks or $>4$ weeks

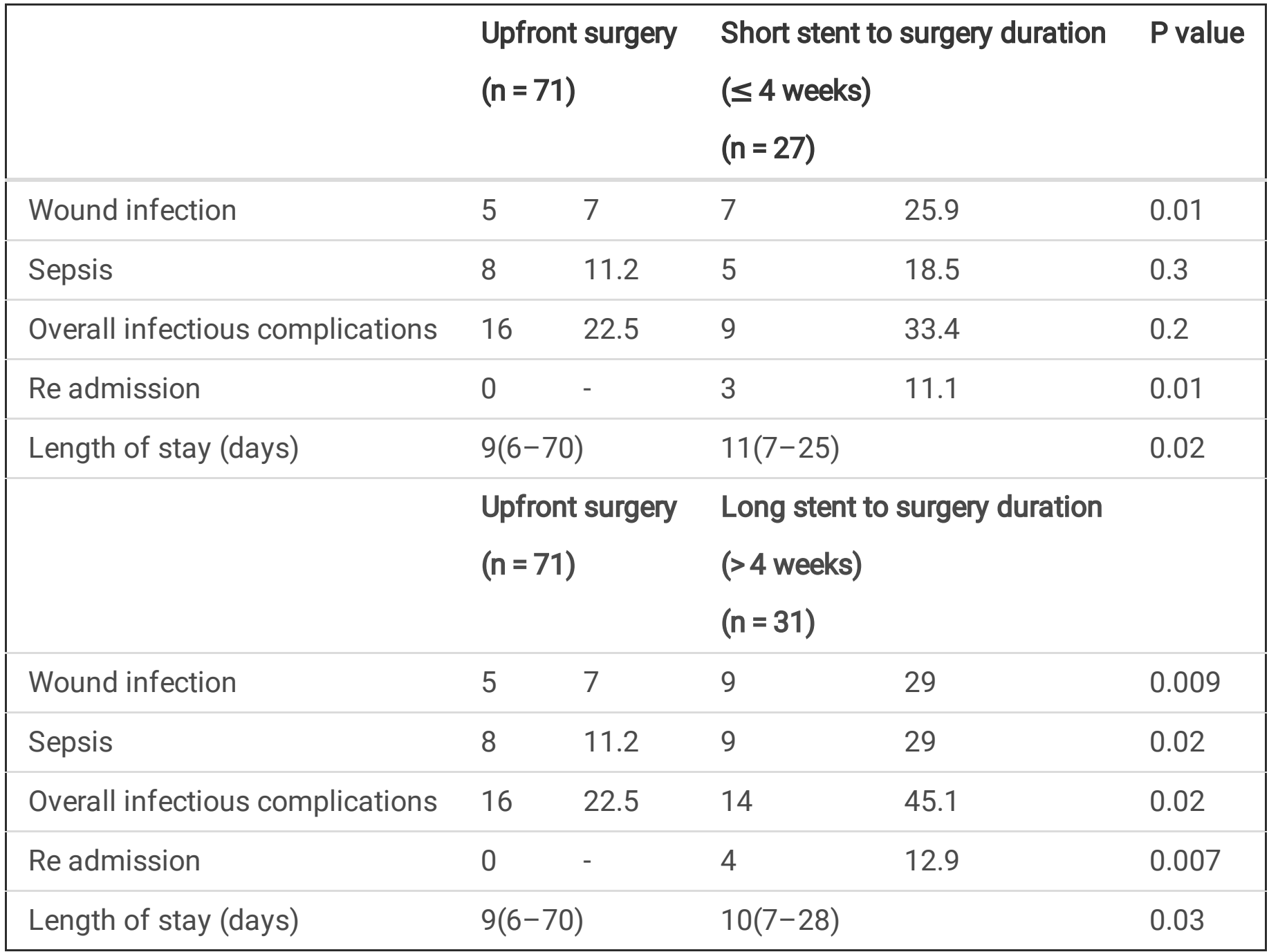

We further explored the rate of infectious complications in stented patients based on 2 weekly stent to surgery intervals. The lowest overall rate of infectious complications was seen when patients were operated within 2 weeks $(22.2 \%)$ or $6-8$ weeks $(12.5 \%)$ of stenting. None of patients in the short stent to surgery duration group had sepsis as shown in Fig. 2. The highest rate of infectious complications was seen between 4-6 weeks after stenting where $60 \%$ patients developed complications.

\section{Discussion}

The current study demonstrates improved outcomes with upfront PD versus PBS in patients with obstructive jaundice. When PBS is performed, patients with short stent to surgery interval appear to have better post operative outcomes. Patients with a stent to surgery interval of 2-6 weeks have the highest rates of infectious complications.

A number of studies have shown increased morbidity in patients with PBS with no impact on mortality [6, 15]. Although a judicious approach to PBS is warranted, there remains a group that could potentially benefit from PBS and a safe stent to surgery duration in this group remains to be determined $[16,17]$. 
Literature on the impact of duration of PBS before PD is limited. These studies have used different statistical designs, variable time period cutoffs with conflicting results. Son and colleagues assessed outcomes in 120 patients who underwent PD after PBS and showed improved outcomes when PD was performed within 2 weeks of PBS [8]. On the contrary, results from a recent multi-center study found a stent to surgery interval < 4 weeks to be associated with frequent postoperative complications [9].

In the current study, we compared outcomes between stented and non stented patients with emphasis upon stent to surgery interval. When compared with patients in the upfront surgery group, patients who underwent PD > 4 weeks after PBS had increased rates of wound infection, sepsis, infected intra abdominal collections, re admissions and prolonged hospital stay. We further assessed outcomes based on 2 weekly intervals in patients with PBS. The rate of infectious complications was lower in patients operated within two weeks or $>6$ weeks after PBS. It's been shown that the risk of poly microbial anaerobic infections increases with indwelling time after ERCP stenting [18]. The risk of stent colonization and obstruction is lower in patients with an indwelling catheter time $<15$ days [19]. This might contribute to lower rate of infectious complications in the short stent to surgery duration group. An element of selection bias cannot be ruled out and it is possible that patients operated $<2$ weeks of stenting achieved superior biliary decompression and had improved nutritional status. Patients with malignant obstructive jaundice are immune compromised. In these patients, there is lowered expression of Interleukin- 6 and $1 \beta$ which appears to be inversely correlated with TB levels. It can take $>4$ weeks after ERCP for TB levels to return to baseline and for immune function to recover [20]. As a result, low risk of infectious complications between 6-8 weeks might reflect recovery of immune function in these patients.

There is no convincing evidence as to which group of patients would clearly benefit from PBS [17, 21]. Variable bilirubin cutoffs ranging from 7.5-14.6 mg/dl, need for neoadjuvant chemotherapy, and unexpected delays in surgery have been considered indication for PBS [17, 22-23]. We are also unaware of the impact of delay in surgery on oncological outcomes after PD [24-25]. In the current study there was no significant difference in median TB levels between stented and non stented patients before PBS, yet outcome variables in non-stented patients were better. These findings suggest that a pragmatic approach should be adopted in decision making as to when PD should be offered after PBS. Patients who achieve adequate biliary decompression early and appear fit to undergo PD should not be made to wait longer unnecessarily. On the other hand, patients with cholangitis, poor nutritional status or unanticipated delays might be offered PD between 6-8 weeks of PBS.

There are certain limitations of the current study. Indications for ERCP and TB cutoffs before PBS in our patient cohort who underwent PD were variable. This was due to a mix referral pattern from within and outside the hospital. For the same reason, complications associated with PBS could not be documented. This however provided a unique opportunity to assess outcomes in actual clinical practice where surgeons frequently see patients after PBS has been performed elsewhere.

\section{Conclusion}


The current study demonstrates favorable outcomes with upfront surgery for PD. PBS should not be used routinely in patients with obstructive jaundice who are potential candidates for PD. When PBS is necessary, PD can be performed early (<2weeks) or late (6-8 weeks) and an individualistic approach to patient's overall heath and disease process should be taken. These results need to be validated prospectively in a larger cohort of patients.

\section{Abbreviations}

PBS Preoperative biliary stenting

PD Pancreaticodoudenectomy

ERCP Endoscopic retrograde cholangio pancreatography

TB Total bilirubin

POPF Post operative pancreatic fistula

\section{Declarations}

Ethics approval and consent to participate: The institutional review board and ethics committee of Shifa International Hospital/Shifa Tameer-e-Millat university approved the study (IRB\# 310-800-2019). A written consent to participate was taken from patients.

Consent for publication: Not applicable

Availability of data and materials: The datasets used during the current study are available from the corresponding author on reasonable request.

Competing interests: The authors declare that they have no competing interests.

Funding: No funding was received.

\section{Authors contributions:}

AHB contributed to concept, design, analysis, drafting and critical review of the manuscript.

RZJ and MKK contributed to data collection, analysis and drafting

FSD contributed to concept, manuscript drafting and critical review

All authors have read and approved the final manuscript.

Acknowledgement: None 


\section{References}

1. Pitt HA, Gomes AS, Lois JF, Mann LL, Deutsch LS, Longmire WP Jr. Does preoperative percutaneous biliary drainage reduce operative risk or increase hospital cost? Ann Surg. 1985;201:545-53.

2. Roughneen PT, Gouma DJ, Kulkarni AD, et al. Impaired specific cell-mediated immunity in experimental biliary obstruction and its reversibility by internal biliary drainage. J Surg Res. 1986;41:113-25.

3. van der Gaag NA, Kloek JJ, de Castro SM, et al. Preoperative biliary drainage in patients with obstructive jaundice: history and current status. J Gastrointest Surg. 2009;13:814-20.

4. Wang C, Xu Y, Lu X. Should preoperative biliary drainage be routinely performed for obstructive jaundice with resectable tumor? Hepatobiliary Surg Nutr. 2013;2:266-71.

5. Aljahdli ES. Preoperativebiliarydrainage in pancreatichead cancerpatients. Saudi J Gastroenterol. 2018;24:139-40.

6. van der Gaag NA, Rauws EA, van Eijck CH, et al. Preoperative biliary drainage for cancer of the head of the pancreas.N. Engl J Med. 2010;362:129-37.

7. Scheufele F, Schorn S, Demir IE, et al. Preoperative biliary stenting versus operation first in jaundiced patients due to malignant lesions in the pancreatic head: A meta-analysis of. current literatureSurgery. 2017;161:939-50.

8. Son JH, Kim J, Lee SH, et al. The optimal duration of preoperative biliary drainage for periampullary tumors that cause severe obstructive jaundice. Am J Surg. 2013;206:40-6.

9. Sandini M, Honselmann KC, Birnbaum DJ, et al. Preoperative Biliary Stenting and Major Morbidity After Pancreatoduodenectomy: Does Elapsed Time Matter?: The FRAGERITA Study Group. Ann Surg. 2018;268(5):808-14.

10. Hafeez Bhatti AB, Dar FS, Sahaab E, et al. Survival advantage with para aortic lymphadenectomy in peri-ampullary cancer: A retrospective cohort study. Int J Surg. 2016;31:58-62.

11. Dar FS, Bhatti AB, Dogar AW, et al. Is pancreaticodoudenectomy with vascular resection a safe procedure in developing country? Early outcomes and review of national literature. Int J Surg. 2015;21:8-13.

12. Singer M, Deutschman CS, Seymour CW, et al. The Third International Consensus Definitions for Sepsis and Septic Shock (Sepsis-3). JAMA. 2016;315(8):801-10.

13. International Study Group on Pancreatic Surgery (ISGPS)

Bassi C, Marchegiani G, Dervenis C, et al; International Study Group on Pancreatic Surgery (ISGPS). The 2016 update of the International Study Group (ISGPS) definition and grading of postoperative pancreatic fistula: 11 Years After.Surgery2016; 161(3):584-591.

14. Clavien PA, Barkun J, de Oliveira ML, et al. The Clavien-Dindo classification of surgical complications: five-year experience. Ann Surg. 2009;250(2):187-96.

15. Fang Y, Gurusamy KS, Wang Q, et al. Meta-analysis of randomized clinical trials on safety and efficacy of biliarydrainage before surgery for obstructive jaundice. Br J Surg. 2013;100(12):1589-96. 
16. Costi R, De Pastena M, Malleo G, et al. Poor Results of Pancreatoduodenectomy in High-Risk Patients with Endoscopic Stent and Bile Colonization are Associated with E. coli, Diabetes and Advanced Age. J Gastrointest Surg. 2016;20:1359-67.

17. Paiella S, De Pastena M, Salvia R. Unmet needs in preoperative biliary stenting for patient candidates for pancreaticoduodenectomy: a viewpoint. Hepatobiliary Surg Nutr. 2019;8:426-7.

18. Basioukas P, Vezakis A, Zarkotou O, Fragulidis G, Themeli-Digalaki K, Rizos S, Polydorou A. Isolated microorganisms in plastic biliary stents placed for benign and malignant diseases. Ann Gastroenterol. 2014;27(4):399-403.

19. Schneider J, Hapfelmeier A, Fremd J, et al. Biliary endoprosthesis: a prospective analysis of bacterial colonization and risk factors for sludge formation. PLoS One. 2014 Oct 14;9(10):e110112.

20. Chowdhury AH, Camara M, Martinez-Pomares L, et al. Immunedysfunction in patients with obstructive jaundice before and after endoscopic retrograde cholangiopancreatography. Clin Sci (Lond) 2016; Sep 1; 130(17): 1535-1544.

21. Perinel J, Adham M.Preoperative biliary drainage for resectable or borderline resectable periampullary tumor: what is the best management?Hepatobiliary Surg Nutr 2019:398-400.

22. De Pastena M, Marchegiani G, Paiella S, et al. Impact of preoperative biliary drainage on postoperative outcome after pancreaticoduodenectomy: An analysis of 1500 consecutive cases. Dig Endosc. 2018;30:777-84.

23. Lassen K, Coolsen MM, Slim K, et al. Guidelines for perioperative care for pancreaticoduodenectomy: Enhanced Recovery After Surgery (ERAS ${ }^{\circledR}$ ) Society recommendations. World J Surg. 2013;37:24058. 10.1007/s00268-012-1771-1.

24. Eshuis WJ, van der Gaag NA, Rauws EA, et al. Therapeutic delay and survival after surgery for cancer of the pancreatic head with or without preoperative biliary drainage.Ann Surg. 2010 Nov;252(5):8409.

25. Matsumoto M, Nakabayashi Y, Fujiwara Y, et al. Duration of Preoperative Biliary Drainage as a Prognostic Factor After Pancreaticoduodenectomy for Pancreatic Head Cancer. Anticancer Res. 2017;37(6):3215-9.

\section{Figures}


$40.00^{-}$

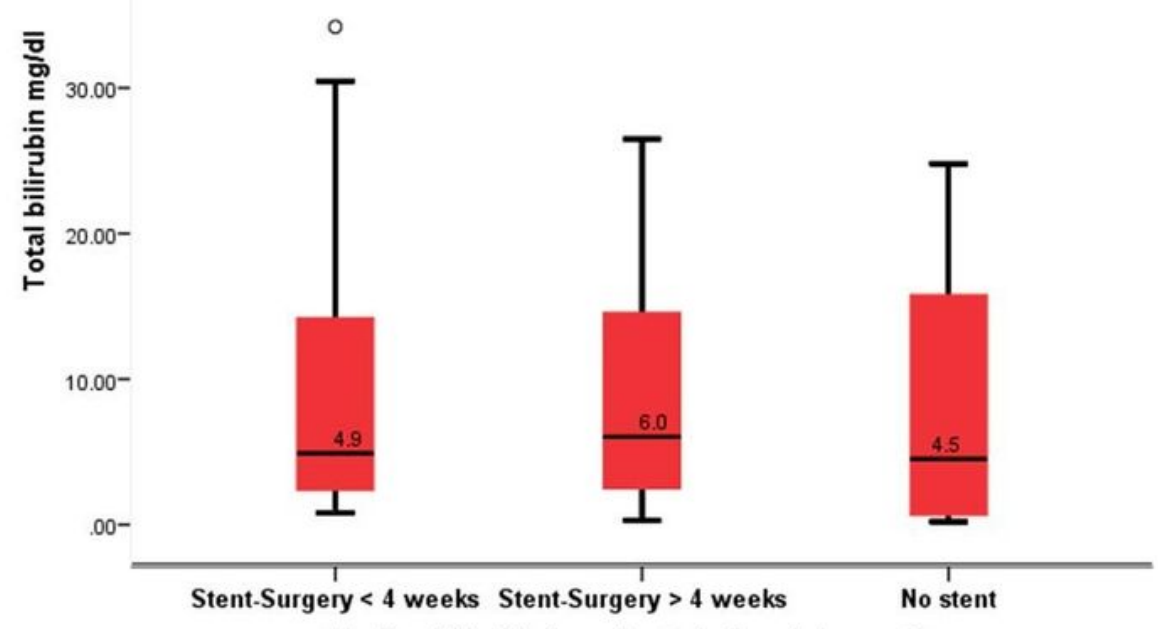

Median bilirubin in patients before intervention

(A)

$40.00-$

30.00-

$10.00-$

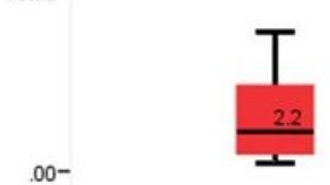

$.00-$

*

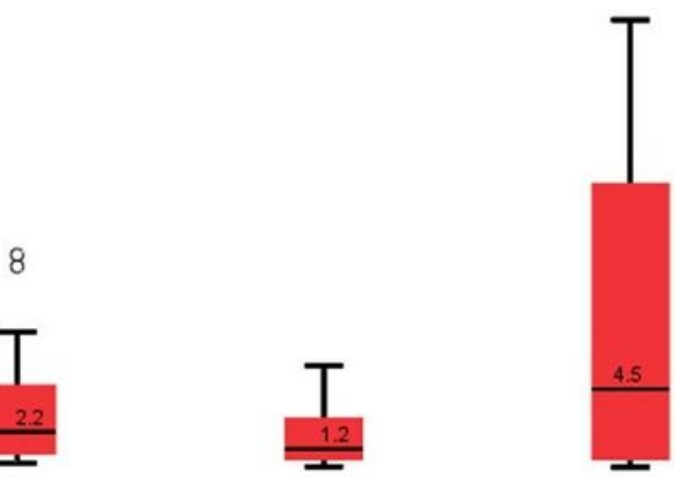

Stent-Surgery $<4$ weeks Stent-Surgery $>4$ weeks

No stent

Median bilirubin before surgery

(B)

\section{Figure 1}

(a) Median bilirubin with interquartile range before any (endoscopic/surgical) intervention (b) after stenting 


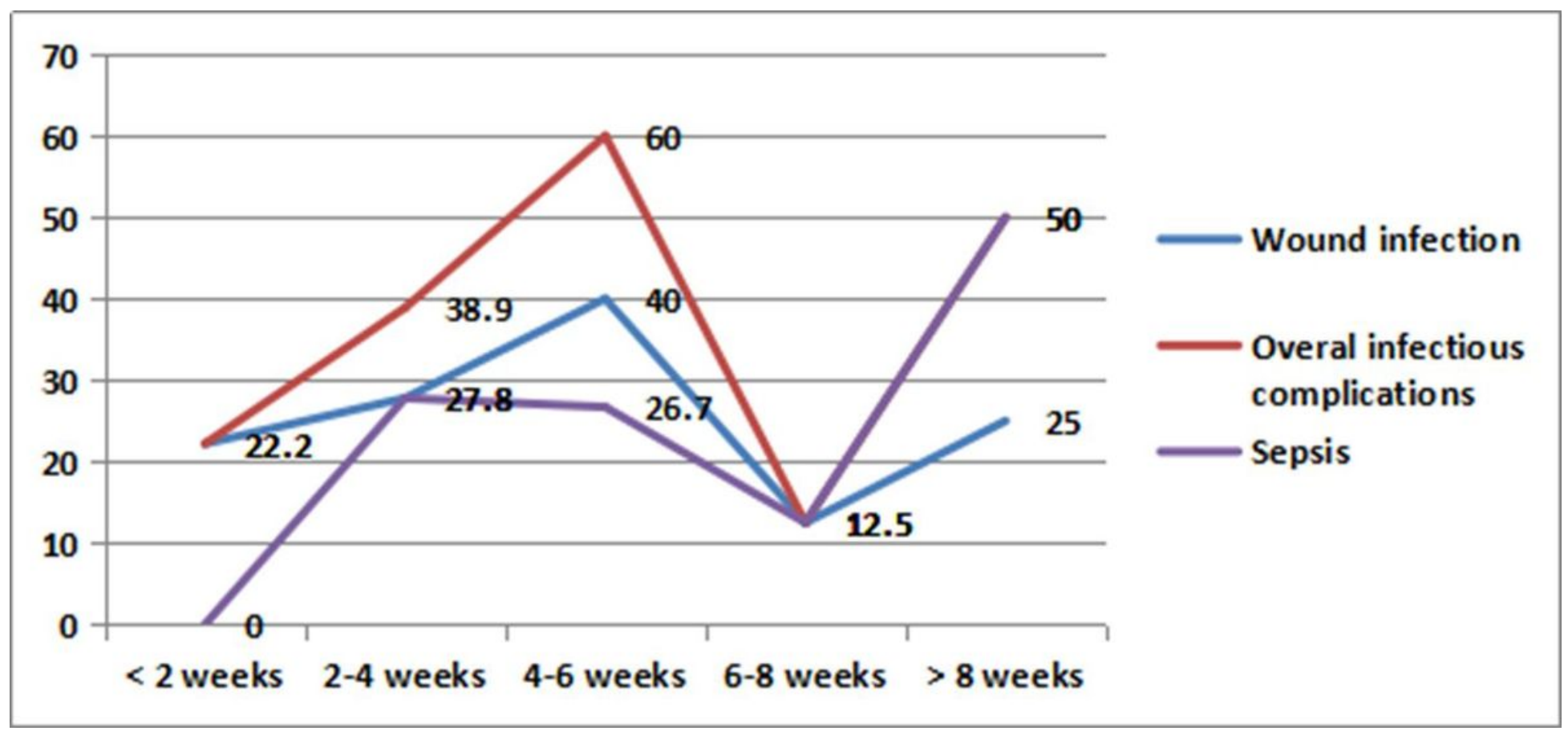

Figure 2

Stent-Surgery duration and percentage of operated patients developing complications during 2 weekly intervals 